\title{
Fiscalité et croissance : Test d'un effet non linéaire au Maroc
}

\section{Taxation and growth: Testing for a non linear effect in Morocco}

\author{
Raid AFTATI $^{1}$ and Abdellah ECHAOUI ${ }^{2}$ \\ ${ }^{l}$ Doctorant en sciences économiques et gestion, Laboratoire d'Analyse Economique et Modélisation, \\ Université Mohammed V de Rabat \\ ${ }^{2}$ Enseignant chercheur en sciences économiques et gestion, Université Mohammed V de Rabat
}

\begin{abstract}
Résumé : L'objectif de cette étude est d'examiner la relation entre la pression fiscale et la croissance économique au Maroc au sens d'Ibn Khaldoun à travers l'estimation du modèle de Scully, du modèle quadratique et de la régression à effet de seuil. Le résultat de notre travail montre que l'interaction entre la fiscalité et la croissance présente une allure non linéaire, sous la forme d'une courbe de Laffer. Cependant les résultats obtenus sont loin d'être concluants. En effet, si notre estimation indique un seuil optimal de taxation qui maximise la croissance au Maroc d'ordre de 24\% les chiffres annoncés par les différents organismes internationaux et nationaux montrent une différence de deux points en moyen par rapport à nos résultats.
\end{abstract}

Mots-clés : Modèle de Scully, Modèle quadratique, Régression a seuil, Pression fiscale.

Abstract: The objective of this study is to examine the relationship between tax pressure and economic growth in Morocco in the sense of Ibn Khaldoun through the estimation of the Scully model, the quadratic model and the threshold effect regression. The result of our work shows that the interaction between taxation and growth presents a non-linear pattern, in the form of a Laffer curve. However, the results obtained are far from being conclusive. Indeed, if our estimate indicates an optimal threshold of taxation that maximizes growth in Morocco of about 24\%, the figures announced by the various international and national organizations show a difference of two points on average compared to our results.

Keywords: Scully model, Quadratic model, Threshold regression, Tax burden. 


\section{Introduction}

La fiscalité est l'instrument privilégié de l'État pour redistribuer les richesses, combler l'écart entre les riches et les pauvres mais aussi un moyen essentiel pour rétablir l'équilibre dans une économie de marché compte tenu de l'asymétrie de l'information .En ce sens, un système fiscal optimal garantit les conditions nécessaires à la justice sociale, l'établissement d'un tel système dépend de nombreux facteurs, dont la taille de pays, le niveau de développement, et d'autres facteurs institutionnels. Plusieurs études affirment que le fait d'augmenter les taxes sur la consommation tout en diminuant les taxes sur le travail et le capital peut stimuler la croissance économique, d'autres études montrent que le fardeau fiscal et la structure fiscale ont un impact significatif sur l'activité économique. À cet égard, Ibn Khaldoun, sur la base de sa théorie de l'État a montré qu'un niveau d'imposition faible est favorable à l'activité économique, à la collecte des recettes et au bien-être économique et social des citoyens. Il a également montré qu'au-delà d'un certain seuil, des taux d'impositions plus élevés entrainent une baisse des recettes fiscales et donc un ralentissement de la production.

La fiscalité est l'un des instruments les plus importants de la politique budgétaire. Cependant, l'effet de la fiscalité sur la croissance économique a été toujours débattu, ni la littérature théorique, ni la littérature empirique ne fournissent des conclusions sur la relation entre la fiscalité et la croissance économique .La question de la détermination de relation entre la fiscalité et la croissance est un exercice difficile à résoudre d'un point de vue pratique car la réalité économique et plus complexe que l'on imagine, sur le plan théorique, il existe plusieurs controverses entre les théoriciens, certains auteurs soutiennent l'idée de l'impact positif ou négatif de la fiscalité sur la croissance ,tandis que, d'autres nuancent cette relation, dans ce sens, l'objectif de ce papier est d'apporter une modeste contribution pour éclairer la nature de cette relation au Maroc en répondant au questionnement suivant : Existe-t-il un effet de seuil de taxation au Maroc? Quel est le niveau optimal de taxation au Maroc ? Pour donner des éléments de réponse à cette problématique, nous présentons d'abord une revue de la littérature théorique et empirique liant la fiscalité et la croissance économique, ensuite nous essayons de quantifier cette relation qui a suscité l'intérêt de plusieurs théoriciens et économistes et qui remonte à chaque fois sur la scène économique et politique à partir d'une batterie de tests statistiques que nous jugeons utiles.

\section{Fiscalité et croissance : Revue de littérature théorique}

La fiscalité optimale reflète le choix de la société entre les objectifs concurrents de l'égalité et de l'efficacité économique, dont le point de départ est de maximiser le bien-être social. Quand une taxe est introduite dans un marché, le premier théorème du bien-être montre que tout équilibre concurrentiel est efficace dans le sens de Pareto. Si l'État ne peut pas observer correctement toutes les caractéristiques 
des individus, son intervention dans un marché créera une distorsion qui déplacera l'équilibre économique vers un état sous optimal.

Les premières études sur la relation entre le taux d'imposition et la croissance remontent au XIVème siècle avec les écrits d'Ibn Khaldoun, il se base sur la théorie de l'État pour affirmer que plus le taux d'imposition est élevé, moins les recettes fiscales seront générer par le gouvernement. Il considère également que les différences de taux d'imposition dépendent des niveaux atteints par une civilisation. Selon lui chaque dynastie passe par cinq phases d'évolutions de la montée jusqu' à décline à chaque niveau correspond un taux d'imposition différent en fonction de l'attitude du chef car il y a forcément des réformes à chaque étape. Dans les premiers stades de la dynastie, les impôts sont peu importants, mais ils génèrent des recettes importantes. Au fil du temps, les dirigeants se succèdent, ils peuvent perdre leurs habitudes tribales au profit d'un État civilisé. Leurs besoins ou demandes augmentent également en partie parce qu'ils sont habitués au luxe. Ainsi, de nouveaux impôts sont imposés à leurs sujets, par conséquent, le taux des anciens impôts est augmenté pour accrôitre leurs revenus. Mais les effets de cette augmentation de la fiscalité sur l'économie se font sentir. Par exemple, les entrepreneurs sont immédiatement découragés. En résultat, la production baisse et les revenus diminuent également. A ce propos Ibn Khaldoun disait : "II faut savoir que, pour ce qui est du prince, sa richesse ne peut s'accroître, si ses revenus devenir abondants, qu'au moyen des ressources de l'impôt. Le développement de celles-ci dépend uniquement de la justice avec laquelle on traite les possédants et des égards qu'on a pour eux : de la sorte, leurs espoirs se donnent libre cours et, le cour joyeux, ils s'empressent de faire produire et fructifier leurs biens. C'est de la sorte que grossit considérablement le revenu du souverain. En dehors de cela, par ses entreprises commerciales, ou agricoles, il ne peut qu'agir de façon rapidement nuisible pour ses sujets, dommageables pour ses finances et fâcheuse pour la culture"

\section{Extrait de l'article de Jalel Berrebeh : la théorie fiscale chez Khaldoun, Revue de Droit 1997, Pub. Faculté de Droit et de Sc.Pol. De Tunis}

Il soutient qu'une charge fiscale lourde et injuste au regard des contribuables car elle entraîne un ralentissement de l'activité économique et un gel de la production, les sujets préfèrent le loisir au lieu de travail. En conséquence, l'assiette fiscale sera limitée et les recettes fiscales diminueront. Pour Ibn Khaldoun il existe un seuil critique au-delà duquel une augmentation de taux d'imposition entraine la réduction des recettes fiscales; en dépassant cette limite, les contribuables préfèrent arrêter ou ralentir la production plutôt que de remettre l'argent dans les caisses de l'État. Résultat, des taux d'imposition élevés récupèrent moins de recettes à l'État, pour garantir un niveau optimal de recettes, il est donc nécessaire de réduire la pression fiscale ou trouver un niveau d'imposition équitable. Cette théorie khaldounienne développée au 4ème siècle a été matérialisée sous la forme d'une courbe par l'économiste Laffer (1981). 
Graphique1 : Courbe de Laffer

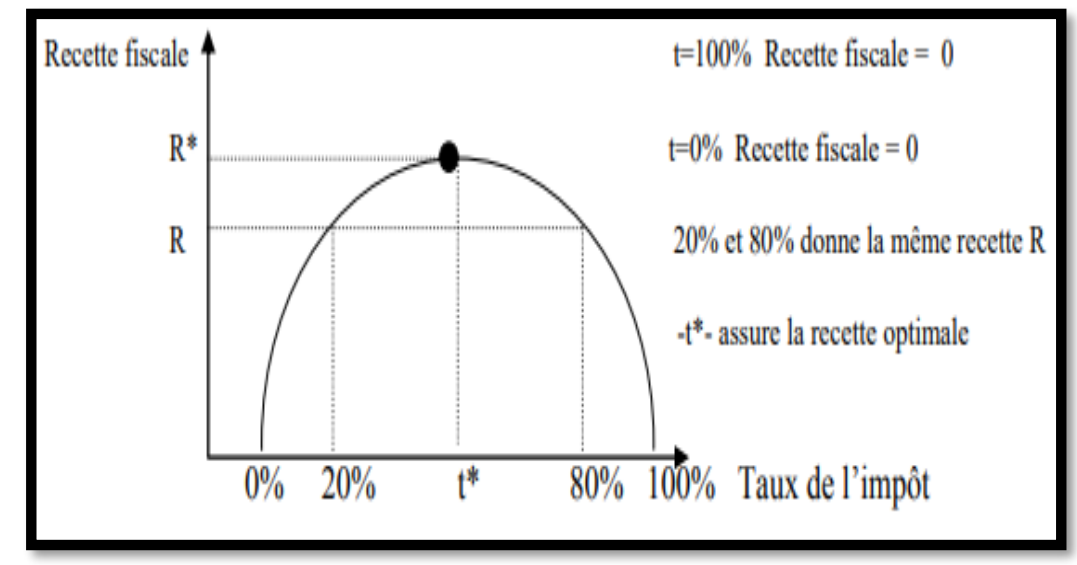

Source : Elaborée par Barro(1990)

Cette courbe indique que l'augmentation des taux d'imposition à un niveau très élevé entraine une diminution des recettes fiscales et une perte de recettes pour l'État. De même, les recettes fiscales augmenteront si le taux d'imposition est réduit. Il existe donc une relation inverse entre le taux d'imposition et les recettes fiscales. Cette relation signifie que le même rendement fiscal $\mathrm{R}$ peut être obtenu avec deux pondérations fiscales différentes, situées des deux côtés (par exemple 20\% et 80\%) du taux d'imposition optimal ( $\mathrm{t}^{*}$ donne les meilleures recettes fiscales, c'est le taux optimal). La forme de la fonction $\mathrm{F}$ impôt/revenu $=\mathrm{F}$ (taux d'imposition) reflète le degré de tolérance fiscale de la nation $\mathrm{R}=\mathrm{F}(\mathrm{t})$.Par conséquent, une réduction des taux d'imposition (de $80 \%$ à $\mathrm{t}^{*}$ ) semble avantageuse pour tout le monde : pour les contribuables, bien sûr, mais aussi pour le gouvernement lui-même, puisque la déclaration de revenus augmente (de $\mathrm{R}$ à $\mathrm{R}^{*}$ avec $\mathrm{R}^{*}>\mathrm{R}$ ). La nécessité de politiques visant à stimuler l'activité économique.

Parmi les théoriciens modernes qui ont abordé le sujet de la taxation optimale. Nous citons Ramsey qui a traité le sujet de la taxation sur la base de la consommation des ménages, et dans la deuxième étude Mirless a pris le sujet de la taxation sur la base du revenu. A ce propos Ramsey(1927) propose la taxation optimale des produits de base «La règle de Ramsey »cette règle est basée sur l'élasticité inverse, selon laquelle la taxation des biens à faible élasticité de la demande à un taux plus élevé réduit la perte d'efficacité, et la taxation des biens à forte élasticité de la demande à un faible taux. La critique la plus importante de cette règle tient au fait que les biens de premières nécessités sont consacrés à la satisfaction des besoins de base qui ont une faible élasticité-prix de la demande et que les biens de luxe ont une élasticité-prix élevée. La taxation des produits de luxe a un taux inférieur à celui des produits de base, en supposant que les consommateurs sont identiques, a une influence négative sur la justice fiscale. Cette situation s'est aggravée avec l'effet de substitution des taxes, ce qui crée une perte d'efficacité, ce problème demeure jusqu'à l'apport de Mirrlees (1971) qui vise la résolution des conflits d'égalité et d'efficacité. Pour lui, une taxation 
plus élevée sur les salaires ne peut plus assurée l'égalité, cependant elle décourage le travail et peut réduire le nombre d'heures des travailleurs.

\section{Fiscalité et croissance : Quelques développements empiriques}

La relation entre la fiscalité et la croissance a fait l'objet de plusieurs études théoriques et empiriques et a suscité l'intérêt des décideurs et économistes. La littérature empirique fournit divers résultats, tels qu'une relation positive, négative, neutre, parfois une relation linéaire ou non linéaire entre la fiscalité et la croissance économique. Nous exposons dans cette partie une revue de littérature empirique qui examine l'effet de la fiscalité sur la croissance économique en distinguant l'effet positif et négatif de la taxation sur la croissance avec présence ou non d'un effet de seuil.

Plusieurs études sur le sujet prêtent un effet négligeable de la fiscalité sur la croissance économique, dans la plupart des modèles de la croissance endogène, la fiscalité n'affecte pas le comportement des individus ou la croissance économique. Arnold. Harberger (1964) a examiné la relation entre la politique fiscale et la croissance économique. Il montre que la fiscalité a un impact très important sur le niveau d'investissement et de la croissance, mais son effet sur la croissance est négligeable sur le long terme. D'autre part, les études qui constatent un effet négatif de la fiscalité sur la croissance économique, nous citons l'étude d'Engen et Skinner (1996) qui ont développé un modèle qui étudie les effets de la taxation sur la croissance économique. Pour ce faire, ils ont utilisé un échantillon de 107 pays durant la période 1970 à 1985 . Ils ont montré que la fiscalité affecte négativement la croissance économique. En 1996, ils montrent à nouveau en s'appuyant sur le modèle de Solow que la fiscalité affecte négativement le niveau de l'activité économique. Cashin(1994) a examiné l'impact de l'investissement public, les transferts publics et les taxes sur le taux de croissance à l'aide du modèle de croissance endogène sur un échantillon de 23 pays développés durant la période 1971 à 1988. Il conclut que la fiscalité a un impact négatif sur la croissance économique. Dans le même sens, Palivos et Yip (1995) ont montré, par l'utilisation du modèle de croissance de type «AK», que les taxes affectent négativement la croissance de long terme. Parmi les études récentes qui révèlent l'impact négatif de la fiscalité sur la croissance, les travaux de Baiardi, Profeta, Puglisi et Scabrosetti (2019) "Fiscal Policy and Economic Growth : Is It Really Important" ils ont indiqué une relation significativement négative entre les recettes fiscales et la croissance économique, l'étude empirique est portée sur les données de panel de21 pays de l'OCDE entre la période de 1971 à 2004 en utilisant l'estimateur PMG. Ainsi, l'étude de Zhao. Z et Lv(2018) utilisent la méthode des variables instrumentales du panel pour étudier les effets des infrastructures et de la fiscalité sur la croissance économique en Chine entre 2002 et 2014. Ils ont montré que les recettes fiscales ont un impact négatif sur la croissance économique sur la période 2008. De meme, le travail de Grdinić, Drezgićet Blažić.H (2017) "Empirical analysis of the relationship between tax structures and economic growth in CEE countries ". Ils ont appliqué l'estimateur PMG à un échantillon de 
20 pays sélectionnés sur la période 1990 à 2010. Ils ont constaté que toutes les formes d'impositions ont un impact négatif sur la croissance économique. En revanche, l'effet positif du niveau de la taxation sur le développement économique est vérifié dans l'étude de A.Kalaš, Mirović, Milenković et Pjanić (2017)"Tax structure and economic growth: Evidence from the European Union" l'étude portée sur un panel de 35 pays de l'OCDE durant la période de 1996 à 2016, les résultats révèlent une relation positive entre la fiscalité et la croissance économique et qu'une augmentation de $1 \%$ des recettes fiscales améliore la croissance économique de 0,29\%.Autre exemple, l'étude de Gashi, Asllani et Boqolli.L(2018) "The effect of tax structure in economic growth" examinent l'effet de la structure fiscale sur la croissance économique au Kosovo au cours de la période 2007à 2015. Ils ont constaté que la plupart des taxes ont un impact positif sur la croissance économique. Par ailleurs, l'étude de Stoilova (2017)"Tax structure and economi cgrowth : Evidence from the European Union " montre d'une façon générale que la structure fiscale est plus propice à la croissance économique. Elle utilise les données de panel regroupées pour la période de 1996 à 2013. De même, le travail de Takumah.Wetlyke.BN(2017)"Links between Economic Growth and Tax Revenues in Ghana: An Empirical Investigation" Ils montrent une relation positive et unidirectionnelle entre les recettes fiscales et la croissance économique. En outre, l'effet non linéaire de la fiscalité en fonction du niveau de développement a été évoquée dans les écrits anciens d'Ibn Khaldoun dans sa théorie de l'État, il défend l'idée de l'effet néfaste de la fiscalité sur l'activité économique et l'évolution des empires, il a montré qu'il existe un seuil au-delà duquel toute forme d'imposition ralentit l'activité économique, cette idée a été matérialisée sous la forme d'une courbe en cloche par Laffer(1981)et fait l'objet de plusieurs études empiriques, A cet effet, nous citons quelques travaux dans ce sens qui traitent une relation non linéaire entre la fiscalité et la croissance, Barro(1990)a montré que l'augmentation du taux d'imposition fournit des ressources importantes pour financer les dépenses publiques, mais au même temps réduit le rendement marginal net du capital privé. Sur la même direction, d'Aydin et Esen (2019) "Optimal tax revenue and economic growth in transition economies : A threshold regression approach» ils ont appliqué un modèle à effet de seuil sur les données du panel dynamique pour examiner une relation non linéaire entre les recettes fiscales et la croissance économique pour un échantillon de 11 pays durant la période de 1995 à 2014. Ils ont montré que le niveau optimal de taxation est d'environ 18\% du PIB pour les économies en pleine transition, 18,5\% pour les économies en développement, et 23\% du PIB pour les économies développées, Ces résultats indiquent qu'il existe un effet de seuil au-delà duquel toute sorte d'imposition pénalise l'activité économique. Enfin, Scully Il a développé un modèle mathématique à l'aide de la fonction de production globale de Cobb-Douglas pour déterminer le seuil d'imposition optimal qui maximise la croissance économique. Pour cela, il suppose qu'il existe deux secteurs : un secteur privé et un secteur public qui produit des biens et des services. Cela lui a permis de mener plusieurs études visant à identifier le taux d'imposition optimal qui maximise la croissance économique aux Etats-Unis et autres pays, le tableau (1) résume certaines études empiriques de scully durant la période allant de 1991 à 2006. 
Tableau1 : Récapitulatif des études empiriques de Scully

\begin{tabular}{|c|c|c|c|}
\hline Modèles & Pays & Période & Taux d'imposition optimal \\
\hline Scully (1991) & 103 pays & 1924 à1987 & $19,3 \%$ du PIB \\
\hline Scully (1995) & États-Unis & 1994 à 1989 & $\begin{array}{l}\text { Un taux d'imposition situe entre } 21,5 \% \text { et } \\
22,9 \% \text { du PIB maximise la croissance aux } \\
\text { États-Unis }\end{array}$ \\
\hline Scully (1996) & $\begin{array}{l}\text { Nouvelle } \\
\text { Zélande }\end{array}$ & 1927 à1994 & $\begin{array}{l}\text { Le taux d'imposition optimal fluctue autour de } \\
21 \% \text { du PIB, avec un taux de croissance annuel } \\
\text { de } 4,7 \%\end{array}$ \\
\hline Scully (1998) & États-Unis & 1950 à 1995 & $21 \%$ du PIB \\
\hline Scully (2003) & États-Unis & 1960 à 2004 & $\begin{array}{l}\text { Le taux d'imposition qui maximise la } \\
\text { croissance est de } 19,3 \% \text { avec une croissance } \\
\text { qui lui associé de } 6.98 \%\end{array}$ \\
\hline Scully (2006) & États-Unis & 1929 à 2004 & $23 \%$ du PIB \\
\hline
\end{tabular}

\section{Source : Auteurs}

Scully montre généralement l'existence d'une relation fonctionnelle entre la croissance économique et la taille du gouvernement dans un pays donné. Il illustre cette hypothèse à partir d'une courbe en U inversé, représentée sur un graphique dont l'axe des abscisses indique les dépenses publiques en pourcentage du revenu national supposées égales aux recettes fiscales en pourcentage du PIB, et l'axe des ordonnées indique le taux de croissance économique.

\section{Estimation du taux de pression fiscale optimal au Maroc}

Dans cet axe, il est proposé d'examiner une relation non linéaire entre la fiscalité et la croissance économique au Maroc, pour laquelle nous allons tester un modèle de Scully et un modèle quadratique puis un modèle à seuil pour confirmer les résultats obtenus sur une période allant de 1980 à 2018. Nous partons de l'observation du graphique qui représente le taux d'imposition (PF) en abscisse et le produit intérieur brut non agricole (PIBN) en ordonnée, un premier regard révèle un effet non linéaire de la fiscalité sur l'activité économique ou une courbe se forme de cloche U. Cela donne l'intuition de l'existence d'un effet de seuil au-delà duquel la fiscalité a un impact négatif sur la croissance économique. 
Graphique2 : Effet non linéaire de la taxation au Maroc

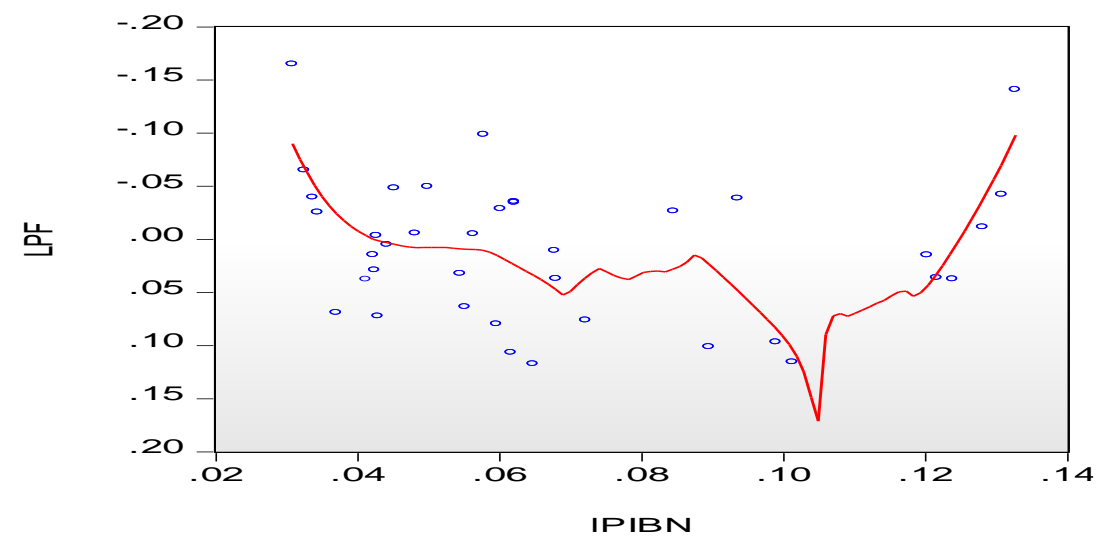

Source : Auteurs, données du MEF

Toutefois, l'observation visuelle ou graphique peut être trempeuse, les travaux empiriques montrent que la détermination du seuil d'imposition optimal n'est pas aisée. L'interaction entre la fiscalité et la croissance pourrait avoir une allure non linéaire, sous la forme d'une courbe de Laffer. A cet égard, Scully a développé un modèle qui permet de terminer le seuil optimal de taxation qui maximise la croissance économique.

\section{A. Modèle de scully}

La relation non linéaire entre la croissance économique et la pression fiscale et examiner empiriquement par scully et auparavant par Laffer (1981).Scully préconise une économie à deux secteurs privé et public. L'État fournit des biens publics finances exclusivement par la contrainte budgétaire $G=\tau \mathrm{Y}$

Avec

$$
\begin{aligned}
& \mathrm{G}: \text { Les dépenses publiques ; } \\
& \mathrm{Y}: \text { Niveau de production ou PIB } ; \\
& \tau: \text { Le taux d'imposition. }
\end{aligned}
$$

Les revenus privés après déduction des impôts $(1-\tau)^{*} Y$ sont utilisés pour produire des biens privés. Les biens publics et privés sont utilisés pour la production nationale et mondiale qui sont présentés à la fonction de production de Cobb-Douglas suivante :

$$
Y_{t}=\alpha\left(G_{t}-1\right)^{a}\left[(1-\tau) Y_{t-1}\right]^{b} \quad \text { eq } 1
$$

En tenant compte des contraintes budgétaires $G=\tau Y$ avec $\mathrm{Y}$ : le produit intérieur brut et $\tau$ : Le taux d'imposition et la transformation de la fonction précédente se forme logarithmique, nous aurons :

$$
\begin{array}{ll}
\operatorname{Ln} Y_{t}=\operatorname{Ln}(\alpha)+a \operatorname{Ln}\left(\tau_{t-1}\right)+\text { b. } \operatorname{Ln}\left[\left(1-\tau_{t-1}\right) Y_{t-1}\right] & \text { eq } 2 \\
\operatorname{Ln} Y_{t}=\delta+a \cdot \operatorname{Ln}\left(\tau_{t-1} Y_{t-1}\right)+\text { b. } \operatorname{Ln}\left[\left(1-\tau_{t-1}\right) Y_{t-1}\right]+U_{t} & \text { eq3 }
\end{array}
$$


Ou a et $b$ représentent des paramètres constants, avec $\alpha>0, a<1$ et $b<1$ et $Y$ : est le PIB et $\mathrm{Ut}_{\mathrm{t}}$ : l'erreur suppose avoir les caractéristiques de bruit blanc. Une différenciation d'eq3 donne l'expression du taux d'imposition optimal comme suit :

$$
\tau^{*}=\frac{\mathrm{a}}{a+b} \quad \text { eq } 4
$$

Au niveau du Maroc, plusieurs études ont été réalisées en appliquant le modèle de Scully, les résultats obtenus sont hétérogènes compte tenu des variables et de la spécification utilisée, dans notre cas l'estimation du taux d'imposition optimal est déterminée à partir de l'équation 3 avec Y représente le produit intérieur brut non agricole(PIBN) et $\tau_{\mathrm{t}}$ représente la pression fiscale dont les cotisations sociales non comprises(PF).

Les résultats de l'estimation du modèle de Scully sont obtenus à partir de l'équation suivante, comme le montre le tableau 2 :

$$
\operatorname{Ln}\left(\mathbf{Y}_{\mathbf{t}}\right)=\mathbf{0 . 4 3 8} * \operatorname{Ln}\left(1-\tau_{\mathbf{t}-\mathbf{1}} * \mathbf{Y}_{\mathbf{t}-\mathbf{1}}\right)+\mathbf{0 . 1 4 2} * \operatorname{Ln}\left(\mathbf{Y}_{\mathbf{t}-\mathbf{1}} * \tau_{\mathbf{t}-\mathbf{1}}\right)+0.75
$$

Tous les coefficients sont significatifs au seuil de risque de $5 \%$, le modèle présente un pouvoir de perdition de $60 \%$, en outre, la statistique de Durbin Watson(DW) est très proche de 2 ce qui prouve l'absence d'autocorrélation des erreurs. Donc le taux de taxation optimal au Maroc en appuyant sur l'équation de Scully est le suivant :

$$
\tau^{*} \frac{b}{b+c}=\frac{0.44}{0.44+0.14}=24.13 \%
$$

\begin{tabular}{|c|c|c|c|c|}
\hline \multicolumn{5}{|c|}{$\begin{array}{l}\text { DependentVariable }: \log (\mathrm{Y}) \\
\text { Method: Least Squares } \\
\text { Sample (adjusted): } 19812018 \\
\text { Includedobservations : } 9 \text { afteradjustments }\end{array}$} \\
\hline Variable & Coefficient & Std. Error & t-Statistic & Prob \\
\hline $\begin{array}{l}\log \left(1-\tau_{\mathrm{t}-1} * \mathrm{Y}_{\mathrm{t}-1}\right) \\
\log \left(\mathrm{Y}_{\mathrm{t}-1} * \tau_{\mathrm{t}-1}\right) \\
\mathrm{C}\end{array}$ & $\begin{array}{r}0.438129 \\
0.142821 \\
0.747512\end{array}$ & $\begin{array}{l}0.100430 \\
0.101184 \\
0.050719\end{array}$ & $\begin{array}{l}0.436253 \\
1.411498 \\
14.73842\end{array}$ & $\begin{array}{l}0.0179 \\
0.0078 \\
0.0000\end{array}$ \\
\hline $\begin{array}{c}\text { R-squared } \\
\text { Adjusted R-squared } \\
\text { S.E. of regression } \\
\text { Sumsquaredresid } \\
\text { Log likelihood } \\
\text { F-statistic } \\
\text { Prob(F-statistic) }\end{array}$ & $\begin{array}{l}0.603567 \\
0.471423 \\
0.102632 \\
0.063201 \\
9.543554 \\
4.567489 \\
0.062303\end{array}$ & \multicolumn{2}{|c|}{$\begin{array}{l}\text { Meandependent var } \\
\text { S.D. dependent var } \\
\text { Akaike info criterion } \\
\text { Schwarz criterion } \\
\text { Hannan-Quinn criter. } \\
\text { Durbin-Watson stat }\end{array}$} & $\begin{array}{c}0.663835 \\
0.141166 \\
-1.454123 \\
-1.388382 \\
-1.595993 \\
1.770305\end{array}$ \\
\hline
\end{tabular}

Tableau 2: Estimation du modèle de Scully

Source : Calculs des auteurs 
L'estimation du modèle fait ressortir un taux d'imposition optimal d'environ 24,13\% au-delà de ce seuil, le niveau d'imposition se répercutera négativement sur l'activité économique, En outre, sur le plan statistique notre modèle semble fiable. En effet le test de normalité d'erreur nous montre que la statistique kurtosis est proche de $3 \%$ et skewness fluctue autour du zéro, donc les erreurs du modèle sont normales.

Graphique 3 : Normalité des erreurs

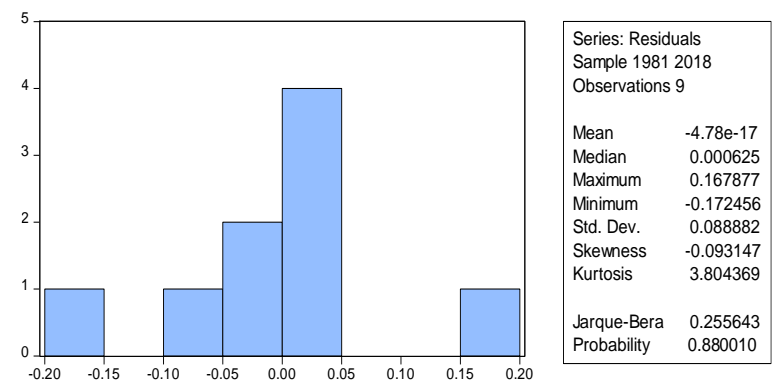

Source : Calculs des auteurs

Dans la même direction, le test de Breusch Pagan est associé à une statistique de Ficher supérieur au seuil du risque de 5\%, donc les erreurs du modèle sont homoscédastiques, de plus l'analyse graphique montre que la variance des erreurs fluctue autour du zéro, par conséquent, notre modèle est validé sur le plan statistique.

Tableau3 : Test Heteroscedasticity Breusch Pagan

\begin{tabular}{|llll|}
\hline F-statistic & 0.389572 & Prob. F(2,6) & 0.6933 \\
Obs*R-squared & 1.034391 & Prob. Chi-Square(2) & 0.5962 \\
Scaledexplained SS & 0.644626 & Prob. Chi-Square(2) & 0.7245 \\
\hline
\end{tabular}

\section{Source : Auteurs}

Finalement, pour tester la stabilité du modèle nous avons opté pour la méthode de CUSUM et CUSUMSq, l'analyse du graphe4 montre que ce sont à l'intérieur de corridor ; au seuil du risque de 5\%, donc notre modèle est stable.

Graphique 4 : Teste de stabilité
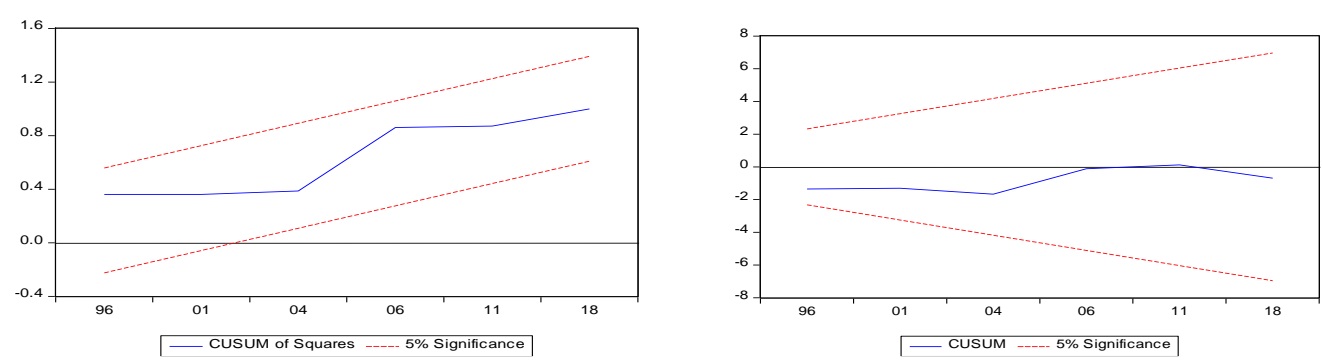

Source : Auteurs 


\section{B. Modèle quadratique}

Le modèle quadratique consiste à détecter une relation non linéaire entre la fiscalité et l'activité économique par le biais des spécifications économétriques tenant compte d'une distribution asymptotique du taux de taxation, cette démarche consiste à estimer des équations quadratiques qui est appuyé sur l'hypothèse d'une courbe sous forme d'une cloche inversée «U», reflète aussi les externalités positives qui auraient le niveau de la taxation sur la croissance économique jusqu'à un seuil au-delà duquel elle devient négative, ainsi nous spécifions une relation entre le niveau de la taxation et la croissance économique par une fonction polynôme du second degré suivant:

Avec

$$
\mathrm{g}_{t}=\theta+\phi \tau_{t}+\phi \tau_{t}^{2}+\vartheta_{t} \quad \text { eq } 1
$$

$\mathrm{g}_{t}:$ Le taux de croissance de PIB ;

$\theta$ : Une constante ;

$\tau_{\mathrm{t}}$ : Le taux de pression fiscale exprimé en pourcentage de PIB ;

$\vartheta_{t}:$ Le terme d'erreur.

Une différenciation d'eq1 nous donne l'expression du taux de la pression fiscale optimale :

$$
\tau_{\text {opm }=-\frac{\varphi}{2 \omega}}^{\text {eq2 }}
$$

Les hypothèses de présence d'un effet de seuil selon le modèle quadratique sont :

- Les signes des coefficients du modèle doivent être opposés ;

- Le premier coefficient traduit l'effet des dépenses publiques sur la croissance doit être positif ;

- Le coefficient associé à la pression fiscale au carré doit être négatif selon Keho (2010) et Anago (2015).

Dans le cas du Maroc, nous allons estimer le modèle quadratique à partir l'équation 3 ci-dessous en introduisant quelques changements dans la spécification du modèle afin de rendre les résultats plus robustes et pour capturer les effets de l'évolution de la structure fiscale dans le temps.

$$
\mathrm{g}_{\mathrm{t}}=\mathrm{Y}_{\mathrm{it}}+\phi \tau_{\mathrm{t}}+\mathrm{w} \tau_{\mathrm{t}}^{2}+\varphi^{\prime} \mathrm{W}_{\mathrm{t}}+\sum \mathrm{Dump}+\varepsilon_{\mathrm{t}} \quad \text { eq3 }
$$

Avec $g_{t}$ : présente l'activité économique, $\tau_{t}$ désigne le taux de taxation exprimé en pourcentage du PIBN, $w_{t}$ :représente l'ensemble des variables de contrôles affectant le taux de croissance économique et $\varepsilon_{t}$ le terme d'erreur. Nous avons pris aussi le PIB hors primaire pour isoler l'effet du secteur agricole, 
les investissements publics (FBCF) et ouverture commerciale (OU) qui sont positivement corrélés avec la croissance économique, puis une variable de contrôle inflation(INF) pour isoler l'effet des prix. Enfin, une variable indicatrice (Dump) permet de capter les chocs affectant le modèle à partir de l'année 2006 période qui coïncide avec le changement de la structure fiscale. La variable indicatrice définie comme suit : $\mathrm{D} 2006=1(\mathrm{t}=2006)$ et 0 si non.

Les résultats de l'estimation du modèle quadratique sont résumés dans le tableau 4, plusieurs tentatives ont été effectuées pour tenir compte des effets des variables indicatrices d'une manière alternative. Le taux de taxation optimale qui permet de maximiser la croissance économique s'obtient par la relation : $\tau_{\text {opm }}=-\frac{\varphi}{2 \omega}$

Tableau 4 : Estimation du modèle quadratique

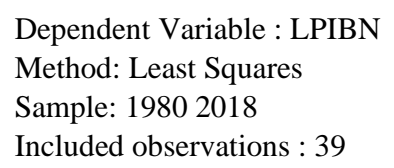

\begin{tabular}{|c|c|c|c|c|}
\hline Variable & Coefficient & Std. Error & t-Statistic & Prob. \\
\hline LPF & $\mathbf{0 . 3 7 0 0 1 4}$ & $\mathbf{0 . 1 3 8 6 4 5}$ & 2.668794 & $\mathbf{0 . 0 1 1 9}$ \\
LPF^2 $_{\text {FBCF }}$ & $\mathbf{- 0 . 0 0 7 6 0 3}$ & $\mathbf{0 . 0 0 3 4 1 0}$ & $\mathbf{- 2 . 2 2 9 4 8 9}$ & $\mathbf{0 . 0 3 2 9}$ \\
OU & -0.060664 & 0.019151 & -3.167697 & 0.0034 \\
INF & 0.027143 & 0.007315 & 3.710739 & 0.0008 \\
DUMP06 & -0.069756 & 0.014346 & -4.862505 & 0.0000 \\
C & 0.383035 & 0.156538 & 2.446907 & 0.0201 \\
& 8.967397 & 1.413176 & 6.345564 & 0.0000 \\
\hline & & & \\
R-squared & 0.940927 & Meandependent var & 12.71297 \\
Adjusted R-squared & 0.929850 & S.D. dependent var & 0.740844 \\
S.E. of regression & 0.196218 & Akaike info criterion & 0.258032 \\
Sumsquaredresid & 1.232049 & Schwarz criterion & 0.040556 \\
Log likelihood & 12.03161 & Hannan-Quinn criter. & 0.150901 \\
F-statistic & 84.94999 & Durbin-Watson stat & 1.418885 \\
Prob(F-statistic) & 0.000000 & \multicolumn{4}{|l}{} \\
\hline
\end{tabular}

Source : Calculs des auteurs

L'output obtenu montre que le niveau d'imposition impact négativement la croissance économique jusqu'à un seuil au-delà duquel l'impact devient positif. Cette hypothèse est vérifiée par les signes des coefficients. En effet, le coefficient associe à la pression fiscale et de signe positif et celui associe à la pression fiscale au carré est de signe négatif. Donc, d'après notre modèle le taux de taxation optimal au Maroc qui maximise la croissance économique est de $24.33 \%$ plus proche au taux annoncé officiellement par CESE et MEF.

$$
\tau_{\text {optm } *}=-\frac{\varphi}{2 \varphi}=-\frac{0.370014}{-2 * 0.007603}=24.33 \%
$$


Sur le plan statistique, notre modèle présente un pouvoir de prédiction plus élevé de $94 \%$, les erreurs issus du modèle suivent un processus de bruit blanc puisque la variance des erreurs fluctue autour du zéro comme il montre le graphe 5. De plus la statistique de ficher associe au test de Breusch Godfrey est supérieur au seuil du risque de $5 \%$ ce qui confirme l'absence d'auto corrélation des erreurs.

Graphique 5 : Normalité des erreurs

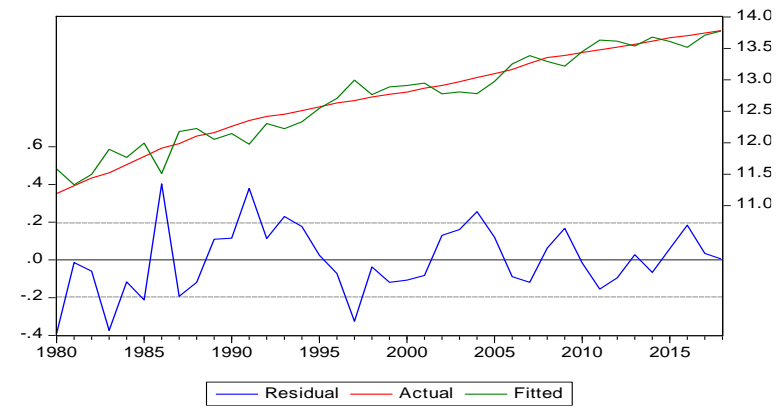

Source : Auteurs

Graphique 6 : Test de stabilité
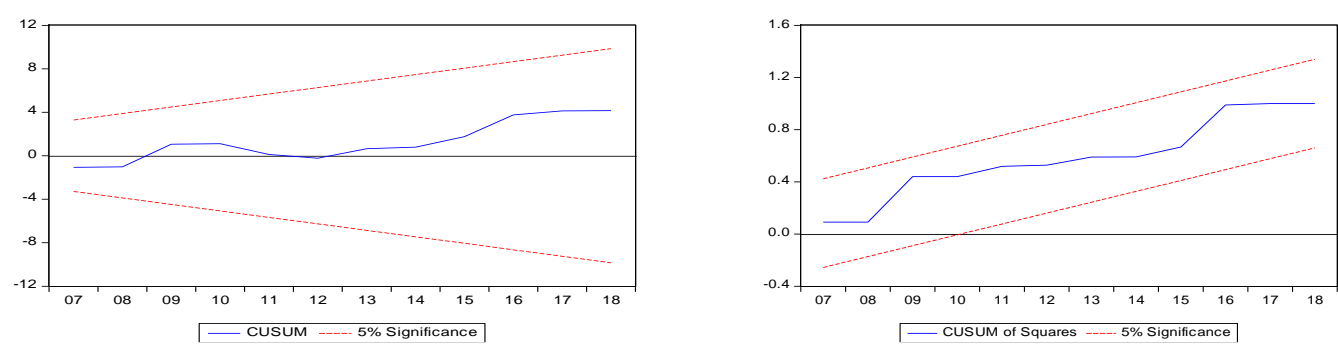

Source : Auteurs

\section{Modèle de régression à seuil}

L'objectif de cette régression est de vérifier l'existence d'un effet non linéaire entre la fiscalité et l'activité économique en utilisant la méthode de régression à seuil discret (TR), ce modèle permet de décrire une forme simple de régression non linéaire comportant des spécifications linéaires par morceaux et un changement de régime qui se produit lorsqu'une variable observée franchit des seuils inconnus.

Les spécifications TR sont très populaires car elles sont faciles à estimer et à interpréter, et capables de produire des non-linéarités intéressantes et une dynamique riche. Pour notre cas la spécification du modèle à seuil passe par la résolution du système d'équation suivant :

$$
\left\{\begin{array}{ccc}
Y_{t}=\alpha_{0}+\alpha_{1} X_{i+} \varepsilon_{i} & \text { si } & q_{t \leq \gamma} \\
Y_{t}=\alpha_{0}+\alpha_{1} X_{i+} \varepsilon_{i} & \text { si } & q_{t>\gamma}
\end{array}\right.
$$

Où

$\mathrm{q}_{\mathrm{t}}$ : Présente la variable de transition ; 
$\mathrm{y}_{\mathrm{t}}:$ La variable explicative ;

$\mathrm{x}_{\mathrm{i}}$ : Vecteur des variables a expliquées ;

$\varepsilon_{\mathrm{i}}:$ Le terme d'erreur.

Étant donné la variable seuil et la spécification de la régression dans l'équation ci-dessus, nous voulons trouver les coefficients $\alpha_{0}$ et $\alpha_{1}$. Nous pouvons également utiliser la sélection de modèle pour identifier le variable seuil, et puisque notre seuil cette fois ci ne prend pas une forme indicatrice donc il suffit d'estimer une seul équation en posant $X_{t}(\gamma)=X t * I t(\gamma)$

$$
Y_{t}=\alpha X t+\alpha_{n} X t(\gamma)_{i+} \varepsilon_{i}
$$

Les variables utilisées dans cette spécification sont semblables à celles utilisées précédemment avec des changements au niveau de la valeur à expliquer nous avons pris la valeur ajoutée industrielle en pourcentage du PIB en utilisant la transformation logarithmique(LVAI) afin de pouvoir obtenir des résultats facilement interprétable, nous avons de même gardé la variable pression fiscale(PF) comme elle a été définie dans les travaux antérieurs, l'intervalle d'étude couvre la période allant de l'année 1980 à 2018.

Tableau 5 : Estimation du Modèle a régression de seuil

Dependent Variable : LVAI

Method:DiscreteThresholdRegression

Sample: 19802018

Included observations : 39

Threshold variable : LPF

\begin{tabular}{|c|c|c|c|c|}
\hline Variable & Coefficient & Std. Error & $\mathrm{t}$-Statistic & Prob. \\
\hline \multicolumn{5}{|c|}{$\mathrm{PF}<16.9582--6$ obs } \\
\hline LPF & 0.323542 & 0.226194 & 1.430374 & 0.1020 \\
\hline $\mathrm{C}$ & 1.063354 & 0.270080 & 3.937185 & 0.0004 \\
\hline \multicolumn{5}{|c|}{$\mathbf{1 6 . 9 5 8 2}<=\mathrm{PF}<\mathbf{1 8 . 9 7 8 8 5}--10 \mathrm{obs}$} \\
\hline LPF & -1.927776 & 0.222444 & -8.666329 & 0.0000 \\
\hline $\mathbf{C}$ & 3.868666 & 0.279557 & 13.83854 & 0.0000 \\
\hline \multicolumn{5}{|c|}{$18.97885<=$ PF --23 obs } \\
\hline LPF & -0.053842 & 0.073177 & -0.735785 & 0.4671 \\
\hline $\mathrm{C}$ & 1.486822 & 0.097368 & 15.27006 & 0.0000 \\
\hline R-squared & 0.804487 & Meandependent var & & 1.428443 \\
\hline Adjusted R-squared & 0.774864 & S.D. dependent var & & 0.027462 \\
\hline S.E. of regression & 0.013030 & Akaike info criterion & & -5.702427 \\
\hline Sumsquaredresid & 0.005603 & Schwarz criterion & & -5.446494 \\
\hline Log likelihood & 117.1973 & Hannan-Quinn criter. & & -5.610600 \\
\hline F-statistic & 27.15742 & Durbin-Watson stat & & 2.433439 \\
\hline Prob(F-statistic) & 0.000000 & & & \\
\hline
\end{tabular}

Source : Calculs des auteurs 
Les résultats obtenus révèlent une relation négative entre la croissance économique et la pression fiscale avec la présence de deux seuils respectivement 16.95 et de 18.97 durant la période d'étude de 1980 à 2018. En outre, nous avons constaté une relation statistiquement significative entre l'activité économique et la pression fiscale au seuil de risque de $10 \%$ lorsque le seuil de la taxation est inférieur au 16.95 , avec une élasticité faible de 0.35 au-delà de cette limite toute sorte de taxation pénalise l'activité économique.

Sur le plan statistique, nous pouvons remarquer que les résidus de notre modèle sont homoscédastiques, en effet, la lecture du graphe7 montre que les erreurs fluctuent auteur de la moyenne zéro.

Graphique7 : Normalité des résidus

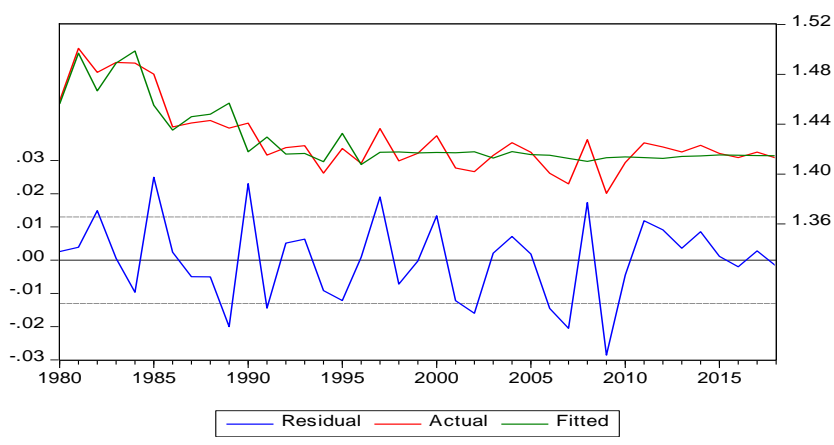

Source : Auteurs

En outre, le teste de Breusch Pagan visant à tester une autocorrélation d'un ordre supérieur à l'unité, indique l'absence d'auto-corrélation des erreurs puisque la statistique associée à Ficher est supérieur au seuil de risque de $5 \%$.

Tableau 6: Test Heteroscedasticity : Breusch Pagan

\begin{tabular}{|lll|}
\hline F-statistic & 1.098137 & Prob. F(5,33) \\
Obs*R-squared & 5.563341 & Prob. Chi-Square(5) \\
Scaledexplained SS & 3.520226 & Prob. Chi-Square(5) \\
\hline
\end{tabular}

Source : Calculs des auteurs

Finalement, le test de stabilité du modèle estime durant la période d'étude, montre que la série de CUSUM et CUSUM Sq est à l'intérieur de corridor ce qui confirme la stabilité de notre modèle.

Graphique 8 : Stabilité du modèle
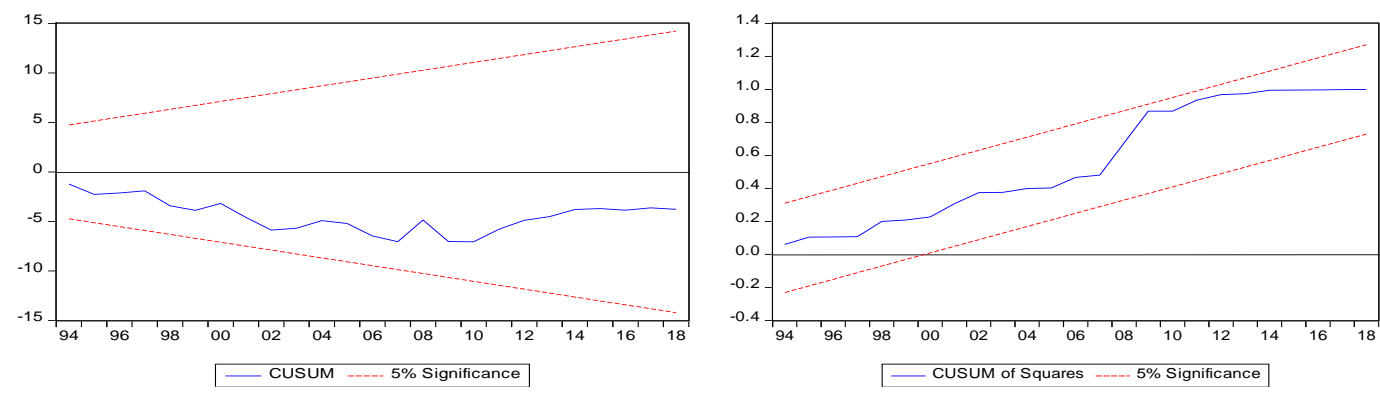

Source : Auteurs 


\section{Conclusion}

Les résultats de notre travail montrent que l'interaction entre la fiscalité et la croissance présente une relation non linéaire, sous la forme d'une courbe de Laffer. Cependant, les résultats obtenus sont loin d'être concluants. En effet, si notre estimation indique un seuil optimal de taxation qui fluctue alentour de $24 \%$ les chiffres annoncés par les différentes instances internationales et nationales montrent une différence de 2 points par rapport à nos résultats. En effet, selon le rapport de l'OCDE(2018).Statistiques des recettes publiques en Afrique. Le niveau d'imposition optimal représente 26,4\% du PIB au Maroc en 2016, soit le troisième ratio le plus élevé en Afrique après la Tunisie 29,4\% et l'Afrique du Sud 28,6 \%.De même, selon le rapport de CESE (2019). Un système fiscal, pilier pour le nouveau modèle de développement. Le ratio recettes fiscales/PIB affiche un taux de 21,15\% en 2017.

En comparaison avec la régression à seuils, les résultats obtenus révèlent que la relation entre la croissance économique et la pression fiscale est caractérisée par la présence de deux seuils respectivement 16.95 et de 18.97 durant la période d'étude 1980 à 2018, soit une différence de 7 points par rapport au résultat de l'estimation du modèle de Scully et du modèle quadratique.

En somme, quel que soit la valeur du taux d'imposition obtenu et la méthode utilisée les résultats empiriques doivent être pris avec méfiance puisque le calcul du taux d'imposition repose sur la notion de pression fiscale celle-ci est sans doute l'un des concepts les plus controversés dans les débats théoriques et empiriques entre économistes et décideurs. Formellement, exprimé sous forme de rapport entre les recettes fiscales et le produit intérieur brut, cet indicateur est d'abord contesté dans sa propre méthode de calcul. Quelles recettes utilisées ? Les recettes de l'État central ou également celles des collectivités locales ? Se limiter à la fiscalité elle-même ou élargir le champ à la parafiscalité ? Quel est le poids du secteur informel dans cette équation ? Au niveau du dénominateur, quel est le rapport impôtPIB ? Quelle est la limite du prélèvement global ?

Finalement, il est difficile d'estimer un niveau optimal de pression fiscale : si cette limite existe indubitablement, il n'est pas aisé de dire où elle se situe ni surtout si elle est déjà atteinte dans un pays donné. Cette question renvoie à des considérations économiques, psychologiques et politiques.

\section{REFERENCES}

[1] Arthur Laffer, (1980). Trop d'impôt tue l'impôt.Université de Chicago L'Amérique.

[2] BARRO, R.J., (1990). Government spending in a simple model of endogenous growth », Journal of Political Economy, 98(5), p. 103-125. 
[3] Gashi.B, Asllani.G, Boqolli. L (2018) .The effect of tax structure in economic growth. Int JEcon Bus Administration 6: 56-6

[4] Ibn Khaldoun (1377).AlMuqadima.

[5] Jalel Berrebeh (1997). La théorie fiscale chez Khaldoun. Revue de Droit Pub. Faculté de Droit et de Sc Pol. De Tunis

[6] Rapport OCDE (2018).Statistiques des recettes publiques en Afrique.

[7] Rapport CESE(2019). Un système fiscal, pilier pour le nouveau modèle de développement.

[8] Scully.G (1998). Measuring the burden of high taxes. Le centre national des analyses politiques

[9] Scully G. (2006). Optimal taxation, economic growth and inequality in the United States. Le centre national des analyses politiques

[10] Scully G.W( 2008). Optimal taxation, economic growth and incomeinequality in the United States.Policy Report No. 316

[11] Scully.G.W(2003).Optimal taxation, economic growth and income inequality. Pub. choice, 115(3/4), 299- 312 